\title{
An engineered cryptic Hxt11 sugar transporter facilitates glucose-xylose co-consumption in Saccharomyces cerevisiae
}

\author{
Hyun Yong Shin ${ }^{1}$, Jeroen G. Nijland ${ }^{1}$, Paul P. de Waal ${ }^{2}$, René M. de Jong ${ }^{2}$, Paul Klaassen ${ }^{2}$ \\ and Arnold J. M. Driessen ${ }^{1 *}$
}

\begin{abstract}
Background: The yeast Saccharomyces cerevisiae is unable to ferment pentose sugars like D-xylose. Through the introduction of the respective metabolic pathway, S. cerevisiae is able to ferment xylose but first utilizes D-glucose before the D-xylose can be transported and metabolized. Low affinity D-xylose uptake occurs through the endogenous hexose (Hxt) transporters. For a more robust sugar fermentation, co-consumption of D-glucose and D-xylose is desired as D-xylose fermentation is in particular prone to inhibition by compounds present in pretreated lignocellulosic feedstocks.

Results: Evolutionary engineering of a D-xylose-fermenting S. cerevisiae strain lacking the major transporter HXT1-7 and GAL2 genes yielded a derivative that shows improved growth on xylose because of the expression of a normally cryptic HXT11 gene. Hxt11 also supported improved growth on D-xylose by the wild-type strain. Further selection for glucose-insensitive growth on D-xylose employing a quadruple hexokinase deletion yielded mutations at N366 of Hxt1 1 that reversed the transporter specificity for D-glucose into D-xylose while maintaining high D-xylose transport rates. The Hxt1 1 mutant enabled the efficient co-fermentation of xylose and glucose at industrially relevant sugar concentrations when expressed in a strain lacking the HXT1-7 and GAL2 genes.

Conclusions: Hxt11 is a cryptic sugar transporter of S. cerevisiae that previously has not been associated with effective D-xylose transport. Mutagenesis of Hxt11 yielded transporters that show a better affinity for D-xylose as compared to D-glucose while maintaining high transport rates. D-glucose and D-xylose co-consumption is due to a redistribution of the sugar transport flux while maintaining the total sugar conversion rate into ethanol. This method provides a single transporter solution for effective fermentation on lignocellulosic feedstocks.
\end{abstract}

Keywords: Sugar transport, Directed evolution, Lignocellulose conversion, Yeast

\section{Background}

Currently, most of the industrial materials and fuels are derived from petroleum that with time will become depleted [1]. Lignocellulosic biomass, the most abundant raw material from hardwood, softwood, and agricultural residues, is considered as an abundant potential

\footnotetext{
${ }^{*}$ Correspondence: a.j.m.driessen@rug.nl

${ }^{1}$ Molecular Microbiology, Groningen Biomolecular Sciences

and Biotechnology, University of Groningen, Zernike Institute

for Advanced Materials and Kluyver Centre for Genomics of Industrial

Fermentation, Nijenborgh 7, 9747 AG Groningen, The Netherlands

Full list of author information is available at the end of the article
}

feedstock for the future production of chemicals and biofuels [2]. Successful conversion of cellulosic biomass into biofuel will require organisms capable of the efficient utilization of xylose, because the free sugar content of plant biomass hydrolysates amounts to $\sim 70 \%$ glucose and $\sim 30 \%$ of xylose [3]. In current fermentation processes, Saccharomyces cerevisiae is the key microorganism for ethanol production as it has the natural capability to convert glucose into ethanol. However, this yeast is unable to ferment xylose because it does not possess a functional xylose dissimilation pathway. Previous research has shown that various metabolic engineering strategies 
can be employed to express both fungal and bacterial genes encoding xylose-utilizing pathways in S. cerevisiae $[4,5]$. A xylose-utilizing pathway in S. cerevisiae has for instance been realized by the introduction of the genes encoding xylose reductase and xylitol dehydrogenase isolated from fungal species [6] or the introduction of heterologous xylose isomerases resulting in a good ethanol yield and productivity during xylose fermentation [7]. Still, in this process, xylose consumption is slow as long as there is residual glucose present in the fermentation broth.

Efficient utilization of xylose is a prerequisite for the large-scale production of biofuels. During pretreatment, inhibitors such as furans, phenols, carboxylic and inorganic acids, aldehydes and alcohols are released from lignocellulosic biomass and these compounds inhibit xylose fermentation $[8,9]$. For instance, acetic acid has a strong and specific negative impact on the xylose consumption rate, which, after glucose depletion, dramatically slows down leaving up to $50 \%$ of the xylose unused [10]. Therefore, growth on xylose in the second phase is more profoundly inhibited by monocarboxylic organic acids compared to growth on glucose. Moreover, engineered $S$. cerevisiae strains show a reduced ethanol tolerance during xylose fermentation as compared to glucose fermentation [11]. Thus, co-fermentation of xylose with glucose would be beneficial for ethanol production from lignocellulosic hydrolysate and a necessity for the development of a robust process.

One of the bottlenecks in the conversion of lignocellulosic biomass to fuels and chemicals is efficient sugar transport [12, 13]. Inefficient transport of pentoses, in addition to low tolerance and throughput, prevent largescale industrial production of next-generation biofuels $[14,15]$. Whereas $S$. cerevisiae contains a multitude of hexose transporters that mediate the uptake of glucose [13], it lacks specific pentose transporters. Instead, pentoses such as xylose enter the cell via the endogenous hexose transporters. This is a sub-optimal process as xylose transport is of low affinity. Importantly, in raw feedstocks, glucose is an abundant sugar and it effectively competes with pentoses for uptake. Engineered strains capable of xylose metabolism indeed first utilize the glucose, and once this sugar is depleted, xylose conversion occurs, resulting in a long fermentation process and an overall slow and incomplete conversion. To improve the xylose uptake activity of $S$. cerevisiae, it is necessary to identify specific transporters mediating the uptake of xylose. One approach is to express specific xylose transporters from other organisms in a xylose-fermenting S. cerevisiae. However, this approach has met little success and only led to minor improvements in the growth rate on xylose and ethanol production [16, 17]. A main problem with heterologous expression of xylose transporters is inefficient targeting to the plasma membrane. Often, overexpressed transporters enter a cellular degradation pathway ending up in vacuoles. A more favorable solution is to employ the yeast endogenous arsenal of transporters and identify those systems with an improved ability to transport xylose. Such transporters would remain connected to the yeast regulatory network that presents different glucose transporters at the cell surface depending on the physiological state of the cells and the glucose concentration in the medium [18].

S. cerevisiae contains at least eight hexose transporters (Hxt) that mediate glucose uptake [19]. Although classified as specific glucose transporters, several Hxt proteins also mediate uptake of xylose albeit with lower affinity [6, 20]. A multiple deletion strain lacking the key transporters Hxt1-Hxt7 and Gal2 was shown to be unable to grow on xylose, while poor growth on glucose was observed [21]. In that study, the remaining Hxt8-Hxt17 transporters were not investigated for xylose uptake as the transcription of their genes is either very low or absent. Nevertheless, these systems present a so far untapped source of transporters with potential xylose transport activity.

Here, we report on an evolutionary engineering approach using chemostat cultivation of a xylose-fermenting S. cerevisiae strain in which the main $H X T 1-7$ and GAL2 transporter genes were deleted. Because of the deficiency in xylose transport, this strain is unable to grow on xylose. However, long-term chemostat culturing allowed cells to adapt and grow on xylose, which could be attributed to the expression of the cryptic HXT11 gene that so far has not been implicated in xylose transport. Further engineering of Hxt11 yielded variants with a dramatically improved specificity towards xylose allowing co-consumption of xylose and glucose at industrial relevant concentrations.

\section{Results and discussion}

Evolutionary engineering of S. cerevisiae strain DS68625 using a chemostat

S. cerevisiae DS68616 is a xylose-fermenting yeast that contains an engineered xylose pathway based on a xylose isomerase and xylulose kinase that shuttle the xylose into the pentose phosphate pathway [22]. A derivative of $S$. cerevisiae DS68616 was constructed in which the HXT17 and GAL2 genes that specify the main hexose transporters were deleted (Table 1). As these hexose transporters are responsible for xylose uptake by the cells, the resultant DS68625 strain is unable to grow on xylose alone (Fig. 1a) $[19,23]$. This strain was used to initiate an evolution experiment in continuous culture under anaerobic condition using xylose as limiting carbon source. Cells 
Table 1 Strains and plasmids

\begin{tabular}{|c|c|c|}
\hline Strain/plasmid & Relevant genotype and/or characteristics & Source or reference \\
\hline \multicolumn{3}{|l|}{ S. cerevisiae strains } \\
\hline DS68616 & $\begin{array}{l}\text { Mat a, ura3-52, leu2-112, gre3::IoxP, loxP-Ptpi:TAL1, loxP-Ptpi::RKI1, loxP-Ptpi-TKL1, loxP-Ptpi- } \\
\text { RPE1, delta::Padh1XKS1Tcyc1-LEU2, delta::URA3-Ptpi-xyIA-Tcyc1, delta::his3 }\end{array}$ & This paper \\
\hline DS68625 & $\begin{array}{l}\text { DS68616, his3::IoxP, hxt2::IoxP-kanMX-loxP, hxt367::IoxP-hphMX-IoxP, hxt145::IoxP-natMX- } \\
\text { loxP, gal2::IoxP-zeoMX-IoxP }\end{array}$ & This paper \\
\hline DS68625-evo & DS68625-derivative after evolutionary engineering & This paper \\
\hline DS71054 & DS71055, glk1::Iox72; hxk1::IoxP; hxk2::Iox72; gal1::IoxP; his3::IoxPnatMXloxP & This paper \\
\hline DS71055 & DS68616-derivative after evolutionary engineering & DSM, The Netherlands \\
\hline \multicolumn{3}{|l|}{ Plasmids } \\
\hline pRS313 & E. coli/yeast shuttle vector; CEN6, ARSH4, HIS3, Amp ${ }^{r}$ & [27] \\
\hline
\end{tabular}
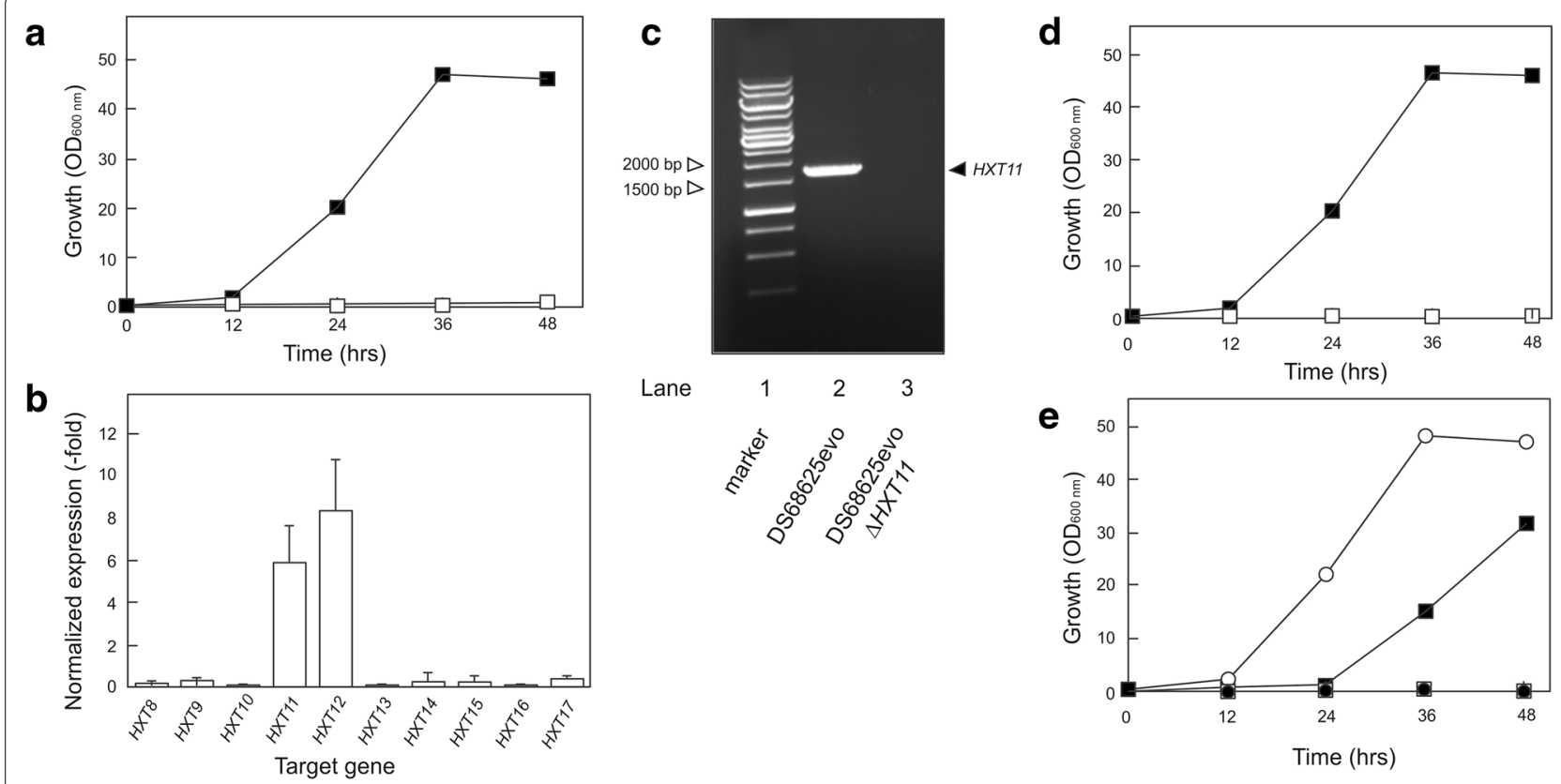

Fig. 1 Utilization of D-xylose by up-regulation of the cryptic Hxt11 hexose transporter. a Growth curve of evolved strain DS68625-evo (filled square) and wild-type DS68625 (open square) on $2 \%$ D-xylose. b Expression level of HXT 8-17 in the strain DS68625-evo. The error bars represent the standard error of the mean from two technical samples. c Deletion of the HXT11 gene from the strain DS68625-evo. d Growth of the DS68625evo (filled square) and $\Delta h x+11$ DS68625-evo (open square) strains on $2 \%$ D-xylose. e Growth of strain DS68616 containing the natively expressed set of Hxt transporters (filled square), DS68625 lacking HXT1-7 and GAL2 but expressing HXT11 (open circle) and HXT12 (filled circle), and the empty vector control (open square) on $2 \%$ D-xylose

were grown at various dilution rate of $0.01-0.05 \mathrm{~h}^{-1}$, and the feed initially contained $2 \%$ xylose and $0.1 \%$ maltose as carbon sources which was changed to $2 \%$ xylose as the sole carbon source after more than $300 \mathrm{~h}$. Maltose was used to support growth of strain DS68625 in the initial stages of the evolution experiment. After $1000 \mathrm{~h}$ ( $\sim 31$ generations), cells capable of growing on $2 \%$ xylose were transferred to shake flask cultures and the single colony isolate growing the most efficiently on xylose was selected. This yielded the evolved strain DS68625-evo that was able to grow on $2 \%$ xylose medium (Fig. 1a), whereas the original DS68625 strain showed no growth on xylose, validating the evolved phenotype.

\section{Expression of HXT genes in the evolved strain DS68625-evo}

Of the 20 members of the $H X T$ gene family, only HXT1-HXT7 are known to encode functional glucose transporter with various affinities for xylose, while HXT8-HXT17 are not expressed when cells are grown in 
the presence of glucose [24]. Previous studies suggested that the remaining $10 H X T$ genes (HXT8-17) are either not expressed or are insufficiently expressed to support growth on xylose $[6,25,26]$. We hypothesized that growth restoration on xylose by the evolved DS68625evo strain is due to the expression of one or more of the cryptic HXT genes (HXT8-HXT17). Importantly, PCR evidence indicated that in the evolved strain, $H X T 1-7$ and GAL2 are still deleted, excluding a contamination of the chemostat experiment (data not shown). The expression patterns of $H X T 8-17$ in the evolved and progenitor strain were compared using cells incubated in a medium containing $2 \%$ xylose. The transcription levels of HXT11 and HXT12 were increased by up to eightfold in the DS68625-evo strain at the beginning of exponential growth phase compared to DS68625, whereas the other HXT genes (i.e., HXT8-HXT10, and HXT13-17) were repressed (Fig. 1b). This suggests that expression of HXT11 and/or HXT12 causes the rescued growth phenotype on xylose.

\section{Hxt11 is responsible for xylose uptake in the evolved strain DS68625-evo}

Hxt11 and Hxt12 are very homologous membrane proteins (i.e., about $98 \%$ ) while HXT12 is considered a pseudo-gene in the S288c genome due to a frame shift mutation [6]. HXT11 and HXT12 were cloned into the low-copy yeast expression vector pRS313-P7T7 under control of the truncated HXT7 promoter [27]. This allows constitutive expression of genes in cells grown on minimal medium containing glucose and/or xylose. The corresponding vectors were transformed into strain DS68625 that is unable to consume xylose because of the aforementioned deficiency in transport. In contrast to HXT12, expression of HXT11 supported growth of strain DS68625 on $2 \%$ xylose (Fig. 1e). Remarkably, growth by the DS68625 strain expressing HXT11 on xylose was even improved relative to strain DS68616 containing the full complement of hexose transporters (Fig. 1e). Furthermore, growth and glucose utilization by the DS68625 strain expressing HXT11 were indistinguishable from that by strain DS68616 (Additional file 1: Figure S1) indicating that HXT11 is an efficient glucose transporter that can support high sugar fluxes. Next, the HXT11 gene was inactivated in the DS68625-evo strain to ascertain that Hxt11 is the key determinant restoring growth of DS68625 on xylose (Fig. 1c). Strain DS68625-evo with the disrupted HXT11 gene was no longer able to grow on xylose medium (Fig. 1d). This demonstrates that the spontaneous growth on xylose of the evolved DS68625evo strain is due to the expression of HXT11.

To validate that growth restoration indeed is caused by increased xylose transport, uptake experiments were performed using $\left[{ }^{14} \mathrm{C}\right.$ - $]$ xylose. As a positive control, the HXT2 gene was overexpressed in strain DS68625, as it has been shown to transport xylose with a reasonable affinity [20]. The strain expressing the Hxt11 transporter indeed showed the effective uptake of xylose with a low $K_{\mathrm{M}}$ of $84.2 \mathrm{mM}$ and but high $V_{\max }$ of $84.6 \mathrm{nmol} / \mathrm{mgDW}$ min (Table 2). The $V_{\max }$ of Hxt11 for xylose uptake was about threefold higher as for Hxt2, while the affinity for xylose uptake was comparable. Compared to Hxt7 and Gal2, the affinity of Hxt11 for xylose uptake was more than twofold higher, while the Vmax of Hxt11 in the same range compared to that of Hxt7 and Gal2. In contrast, the affinity of Hxt11 for glucose is much lower than that of Hxt7 and Gal2 (Table 2) which makes Hxt11 an ideal scaffold for further engineering of its xylose specificity. In this respect, both with Hxt11 and Hxt2, $\left[{ }^{14} \mathrm{C}\right.$ - $]$ xylose uptake was strongly inhibited by glucose, but xylose transport by the Hxt11 transporter appeared less sensitive to glucose inhibition (Additional file 1: Figure S2).

\section{Genomic analysis of evolved strain DS68625-evo}

The genome of the evolved strain DS68625-evo was sequenced and compared with that of the progenitor

Table $2 K_{m}$ and $V_{\max }$ values for D-glucose and D-xylose uptake by Hxt11 transporters expressed in strain DS68625

\begin{tabular}{|c|c|c|c|c|c|c|}
\hline \multirow[t]{2}{*}{ Transporter } & \multicolumn{2}{|l|}{$K_{\mathrm{m}}(\mathrm{mM})$} & \multirow[t]{2}{*}{$K_{\mathrm{m}} \mathrm{Glc} / \mathrm{Xyl}$ (ratio) } & \multicolumn{2}{|c|}{$V_{\max }(\mathrm{nmol} / \mathrm{mg} \mathrm{DW} \min )$} & \multirow[t]{2}{*}{ Source } \\
\hline & Glucose & Xylose & & Glucose & Xylose & \\
\hline Hxt11 & $33.4 \pm 2.1$ & $84.2 \pm 10.0$ & 0.40 & $156.4 \pm 7.6$ & $84.6 \pm 2.4$ & This study \\
\hline Hxt11-N366D & $87.0 \pm 6.4$ & $106.7 \pm 21.7$ & 0.82 & $197.8 \pm 11.4$ & $86.5 \pm 2.0$ & This study \\
\hline Hxt11-N366T & $194.4 \pm 47.9$ & $46.7 \pm 2.7$ & 4.16 & $238.6 \pm 7.4$ & $76.2 \pm 4.8$ & This study \\
\hline Hxt11-N366 M & $144.9 \pm 36.0$ & $50.1 \pm 9.7$ & 2.89 & $143.0 \pm 17.2$ & $65.0 \pm 6.8$ & This study \\
\hline $\mathrm{Hxt2}$ & n.d. & $51.2 \pm 0.1$ & n.d. & n.d. & $23.8 \pm 0.4$ & This study \\
\hline Gal2 & $1.5 \pm 0.2$ & $225.6 \pm 15.8$ & 0.01 & $27.2 \pm 0.9$ & $91.3 \pm 3.2$ & {$[36]$} \\
\hline Hxt7 & $0.5 \pm 0.1$ & $200.3 \pm 13.2$ & 0 & $26.0 \pm 1.1$ & $67.0 \pm 2.0$ & {$[36]$} \\
\hline
\end{tabular}

n.d. not determined, \pm standard deviation with $n=2$ 
DS68625, resulting in the identification of 80 singlenucleotide polymorphisms (SNPs) in the DS68625-evo strain (Additional file 2: Table S1). To access which of the mutations is preliminary responsible for the changed expression level of the HXT11 gene, we focused on the SNPs that are related to HXT regulation. Of the 80 SNPs that resulted in amino acid changes, two are in genes that are potentially involved in such regulation. Gene SIZ1 which encodes a small ubiquitin-like modifier (SUMO) is an important component of the regulation of the Snf1 function as it interacts with the SUMO-interacting sequence motif located near the active site of Snf1, which inactivates Snf1 protein directing it for destruction via the Slx5-Slx8 (SUMO-directed) ubiquitin ligase [28]. In the evolved strain, SIZ1 carries two amino acid mutations, Asn531Tyr and Asn534Lys, which are not in a functionally defined region. In addition, a frame shift mutation was identified in gene HAP4 at nucleotide 1619. Hap4p is a DNA-binding protein that is part of a multimeric HAP complex I that binds to the consensus $5^{\prime}$-TNATTGGT-3' sequence in the promoter of target genes $[29,30]$ and affects gene expression. Possibly, these mutations contribute to the deregulation of HXT11 gene expression observed in the evolved strain.

The exact mechanism how HXT11 expression is induced in the evolved strain is unknown. As confirmed by genomic DNA sequencing, the nucleotide sequence of the promoter region of HXT11 in the DS68625-evo strain was indistinguishable from that in the original DS68625 strain. Recently, it was reported that the expression of HXT11 is positively regulated by the Pdr1p and Pdr3p transcriptional factors and that expression is not induced by glucose [31]. In addition, in xylose-grown cells, the genes encoding the enzymes of the citric acid and glyoxylate cycle, respiration and gluconeogenesis showed higher expression levels than in glucose repressed cells [32]. These genes are regulated by the transcriptional regulators ADR1, CAT8, HAP4, SIP1-2 and -4, and REG2 [33]. It seems that expression of $H X T 11$ is regulated differently from the main $H X T 1-7$ transporter genes, but the mechanism underlying expression of HXT11 in the evolved strain is complex and likely not due to single mutations.

\section{Engineering of Hxt11 for improved xylose transport in the presence of glucose}

To improve on the xylose transport specificity of Hxt11, the HXT11 gene was mutagenized by error-prone PCR and subjected to a screen for improved xylose transport in S. cerevisiae in the presence of a high concentration of glucose. Herein, a strain was constructed that is unable to grow on D-glucose by deleting hexokinase activity-encoding genes in a strain that contains an engineered D-xylose metabolic pathway and therefore grows on D-xylose. The three hexokinases Hxk1p, Hxk2p and Glk1p differ in their affinity and specificity for glucose and ATP [34] and all phosphorylate glucose to glucose-6-phosphate. However, in an earlier study, the triple hexokinase deletion strain continued to grow on glucose after several rounds of evolutionary engineering, and re-sequencing of this evolved strain revealed a mutant GAL1 sequence that rescued glucose phosphorylation in the triple hexokinase deletion strain thereby re-enabling growth on glucose [35]. Therefore, to enable a stable screening platform, four hexokinase genes, GLK1, HXK1, HXK2 and GAL1, were deleted from S. cerevisiae strain DS71055 to block glucose consumption. The corresponding quadruple hexokinase deletion strain DS71054 (Table 1) is unable to grow on D-glucose but still grows on D-xylose (Additional file 1: Figure S3) and this growth phenotype did not revert when cells were exposed to xylose and glucose for longer periods of time (data not shown). The DS71054 strain is similar to the S. cerevisiae strain used by Farwick and coworkers [36], except that in the latter study, the putative hexokinase gene $Y L R 446 W$ was deleted instead of GAL1. In a previous study, YLR446 W was found not to be responsible for the remaining hexokinase activity in a triple $\Delta h x k 1, \Delta h x k 2, \Delta g l k 1$ deletion strain [35].

A library of mutagenized HXT11 genes was cloned into the yeast vector pRS313-T7P7 and transformed into strain DS71054. Three thousand clones were screened for growth on xylose $(1 \%)$ in the presence of a 15 -fold excess of glucose using a 96-well plate format. This screen yielded eight mutants that showed faster growth than the strain containing the wild-type Hxt11 transporter. The corresponding plasmids were isolated and re-sequenced. All eight plasmids contained the HXT11 gene with the same mutation leading to a change at position 366 from an asparagine $(\mathrm{N})$ into an aspartate (D) residue. When the Hxt11-N366D mutant was expressed in strain DS71054, it also displayed faster growth on $1 \%$ xylose in the presence of a 15-fold excess of glucose compared to wild-type Hxt11 (Fig. 2a). When expressed in strain DS68625, Hxt11 and Hxt11-N366D showed a similar growth rate on xylose (Additional file 1: Figure S4), indicating that the mutation primarily affects the ability of glucose to compete with xylose transport, rather than improving xylose transport per se. Indeed, the affinity for glucose uptake by Hxt11-N366D was reduced 2.8fold compared to the wild-type Hxt11, while there was only a negligible effect of the mutation on the xylose uptake affinity (Table 2). The $V_{\max }$ for glucose uptake by Hxt11-N366D was slightly increased compared to Hxt11, while the $V_{\max }$ for xylose uptake remained unchanged (Table 2). Because of these altered kinetic parameters, xylose uptake by the Hxt11-N366D mutant was notably 

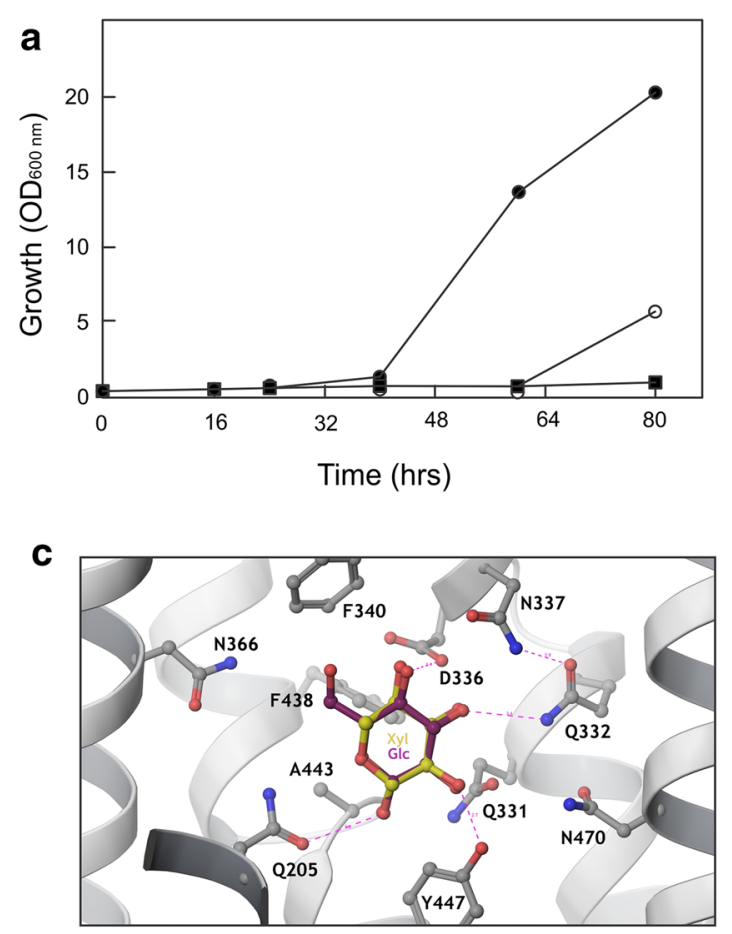
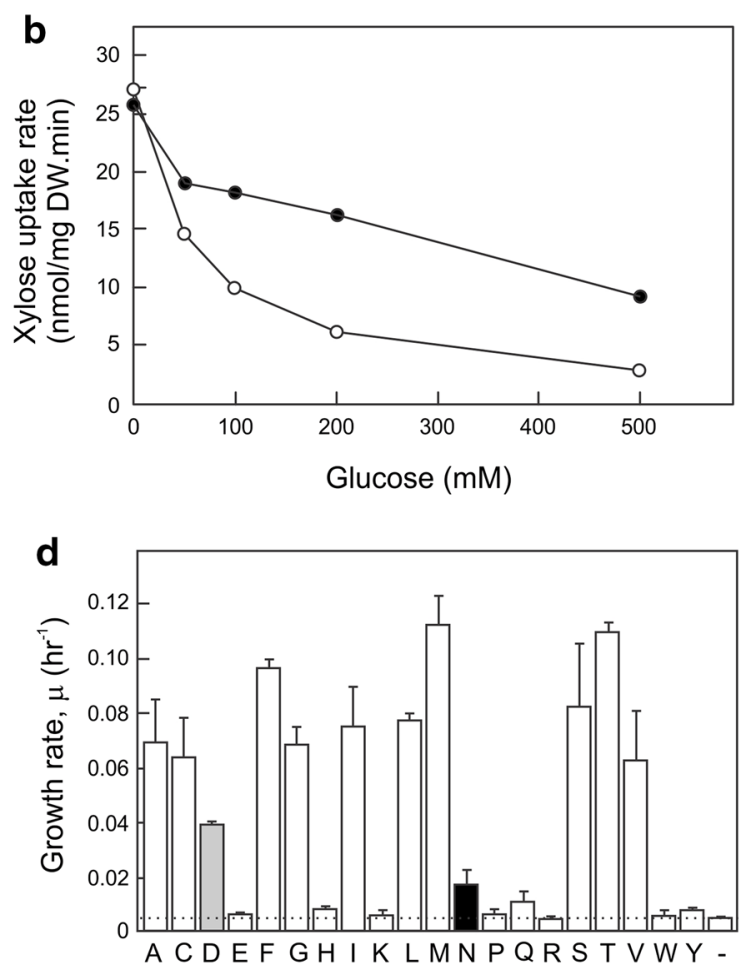

Amino acid at position 366

Fig. 2 Characterization of the N366X mutants of HXT11. a Growth of the hexokinase deficient DS71054 strain containing the empty vector (filled square), or expression vectors bearing HXT11 (open circle), and HXT11-N366D (filled circle) on $1 \%$ xylose and $15 \%$ glucose. b Uptake of [ $\left.{ }^{14} \mathrm{C}-\right]$ xylose by the DS68625 strain expressing HXT11 (open circle) and HXT11-N366D (filled circle) in the presence of increasing concentrations of glucose. c Detailed view of the sugar-binding pocket of the Hxt11 homology model, showing the first shell amino acid side chains that interact with bound glucose (cyan) and xylose (yellow). N366 is located to the left, pointing the side chain towards the 6-OH and 6- $\mathrm{CH}_{2}$ of glucose. Most residues in this pocket are strictly conserved between Hxt11 and XylE, apart from D337 (I in XylE), A442 (G in XylE), Y446 (W in XylE) and N469 (Q in XylE). The scheme was constructed using Maestro (Schrödinger LLC, NY, USA). d Exponential growth rates ( $\mu$ ) of strain DS71054 expressing the indicated Hxt11-N366X mutants when grown on $10 \%$ glucose and $1 \%$ xylose. The black bar indicated the wild-type position, and the gray bar indicates the N366D mutant obtained in the error-prone mutagenesis. The dashed line indicates the growth rate of strain DS71054 without any introduced transporter. The error bars are the standard error of the mean from two technical samples

less sensitive to glucose inhibition as compared to the wild-type Hxt11 (Fig. 2b).

To understand the molecular mechanism of the reduced affinity for D-glucose by the N366D mutation in Hxt11, a homology model for Hxt11 was constructed based on the crystal structure of XylE from E. coli with xylose bound in the occluded state (PDB ID: 4GBY) [37]. Notably, N366 is predicted to be located in a conserved region in transmembrane domain (TMD) 8 between Hxt11 and XylE, which has the sequence 363-GVVN366 in Hxt11 and 322-GVIN-325 in XylE, where the conserved asparagine residue is marked in bold (Fig. 2c). Therefore, like N325 in the crystal structure of XylE, N366 in Hxt11 is in close proximity to the $5-\mathrm{CH}_{2}$ of the ring form of $\mathrm{D}$-xylose. In the case of a bound D-glucose in Hxt11 (Fig. 2c), the $6-\mathrm{CH}_{2}$ and $6-\mathrm{OH}$ would point towards N366, which upon mutation to an aspartate apparently lowers the affinity for D-glucose, while leaving D-xylose binding undisturbed. It is unclear why such a subtle change in the charge and not size of the amino acid side chain results in such a large effect on the affinity for D-glucose, especially in view of the lack of a direct interaction between the xylose $5-\mathrm{CH}_{2}$ and modeled glucose 6- $\mathrm{CH}_{2}$ and 6-OH in XylE and the Hxt11 model. The answer may lie in the interactions between the sugar and amino acids in the outward open or inward open state of the transporter, for which co-crystal structures are as of yet unavailable.

To examine the mutational space, N366 was also replaced with each of the other 18 remaining amino acids to generate a series of N366X mutants. The corresponding genes were expressed in the hexokinase deficient 
strain DS71054 and evaluated for their ability to utilize $1 \%$ xylose in the presence of $10 \%$ glucose using 96-well plates. Growth on xylose in the presence of glucose was improved when N366 was substituted by a methionine (M) or threonine (T) residue (Fig. 2d), and was up to threefold improved compared to the N366D substitution. Amino acids with a positively charged $(\mathrm{K}, \mathrm{R})$, polar $(\mathrm{Q}, \mathrm{H})$ or bulky hydrophobic side chains (W, Y except for F) did not support growth on xylose under the screening condition, whereas in particular aliphatic side chains (L, I, V, $\mathrm{M})$ or polar (S, T) supported enhanced growth on xylose in the presence of glucose. The mutants were also tested for growth on glucose when expressed in strain DS68625. The N366M and N366T Hxt11 mutants showed similar growth on glucose or on xylose as compared to the wildtype Hxt11 (Additional file 1: Figure S4). In addition, the expression level of all individual mutants was determined using GFP-tagged Hxt11 proteins (Additional file 1: Figure S5). All Hxt11-GFP proteins were highly expressed on the plasma membrane, except for the N366W and N366Y mutant Hxt11 proteins that showed a cytosolic and likely vacuolar localization of the fluorescence. This suggests protein misfolding and/or faulty targeting of these mutants and explains the low activity in the growth experiments with xylose and glucose.

Compared to the wild-type Hxt11, the affinity for glucose transport by the N366T and N366M Hxt11 mutants was reduced up to five- and fourfold, respectively (Table 2). In contrast, the affinity for xylose uptake by these mutants increased up to twofold relative to Hxt11 causing an overall tenfold improvement of the transporters specificity for xylose versus glucose. Strikingly, these mutant transporters exhibit a higher affinity for xylose transport compared to glucose, while the $V_{\max }$ for xylose uptake remained largely unchanged relative to Hxt11. These data demonstrate that the N366T and N366M mutations convert Hxt11 into a transporter with a greater specificity for xylose than for glucose.

All hexose transporters in $S$. cerevisiae including Hxt $11 \mathrm{~m}$ belong to the major facilitator superfamily (MFS) [42]. They share a high identity in the transmembrane region but differ substantially in size and amino acid sequence of their $\mathrm{NH} 2-$ and $\mathrm{COOH}$-terminal region. It was previously reported that in a chimeric transporter of Hxt1 and Hxt2, N331 in TMD 7 is a key residue responsible for the moderately high-affinity glucose transport activity of Hxt2 [43]. In addition, N340 of Hxt7, which corresponds to N331 of Hxt2 is a key residue that determines the glucose affinity in Hxt7, suggesting that the mechanism of substrate recognition is similar in Hxt7, Hxt2 and Hxt1 [44]. Also, the Gal2-N376V and Hxt7-N370S mutants that localize to TMD 8 were shown to be equipped with an improved affinity for D-xylose
[28]. The latter residue corresponds the residue in Hxt11 as reported in this study lending further support for the notion that TM8 is crucial for substrate specificity.

\section{Co-fermentation of glucose and xylose by the mutant HXT11-expressing strain DS68625}

Because of the marked effects of the N366 mutations in Hxt11 on the sugar specificity, mutants were further examined for their ability to co-metabolize xylose and glucose under industrial relevant sugar concentrations, i.e., $7 \%$ glucose and $4 \%$ xylose under anaerobic conditions which is experimentally an usually stringent condition for co-metabolism validation as other studies use equimolar concentrations. N366M Hxt11 (Fig. 3c) and in particular N366T Hxt11 (Fig. 3d) supported a near to perfect co-consumption of glucose and xylose when expressed in strain DS68625, except for the early stages of fermentation at the high glucose concentrations where glucose metabolism was slightly preferred. Effective co-consumption was also observed when the mutants were grown at equal glucose and xylose concentrations, i.e., $1.0 \%$ each (Additional file 1: Figure S6). Compared to the the wild-type Hxt11 (Fig. 3b) and the DS68616 strain containing the full complement of hexose transporters (Fig. 3a), co-consumption is due to reduced and increased rates of glucose and xylose conversion, respectively. Importantly, the wild-type Hxt11 (Fig. 3b) and the DS68616 strain (Fig. 3a) showed a severely delayed consumption of xylose which is typically observed in cometabolism events.

For further analysis, the specific xylose consumption rates were measured during the anaerobic cultivation performed in the presence of $7 \%$ glucose. The specific xylose consumption rate of strain DS68625 with Hxt11 N366T was improved to $0.63 \mathrm{~g} / \mathrm{g}$ cell h compared to 0.49 and $0.54 \mathrm{~g} / \mathrm{g}$ cell $\mathrm{h}$ for strain DS68616 and strain DS68625 expressing Hxt11p, respectively. On the other hand, the specific glucose consumption rate with Hxt11 N366T was reduced to $1.23 \mathrm{~g} / \mathrm{g}$ cell $\mathrm{h}$ compared to $1.74 \mathrm{~g} / \mathrm{g}$ cell $\mathrm{h}$ for Hxt11p (Additional file 2: Table S2). Additionally, the xylose consumption rate in the presence of various concentrations of glucose was improved up to twofold for strain DS68625 carrying the Hxt11 N366T and N366M mutants as compared to the same strain with Hxt11 or strain DS68616 (Additional file 1: Figure S7). The ethanol productivity of these improved DS68625 strains was only slightly lower compared to strain DS68616 but the ethanol yield was the same (Additional file 2: Table S2). Overall, these data demonstrate that the mutagenesis of the N366 position of Hxt11 yields mutants that as a single transporter mediates a balanced uptake of glucose and xylose, thereby supporting co-consumption of the these sugars at overall sugar conversion rates that are in 

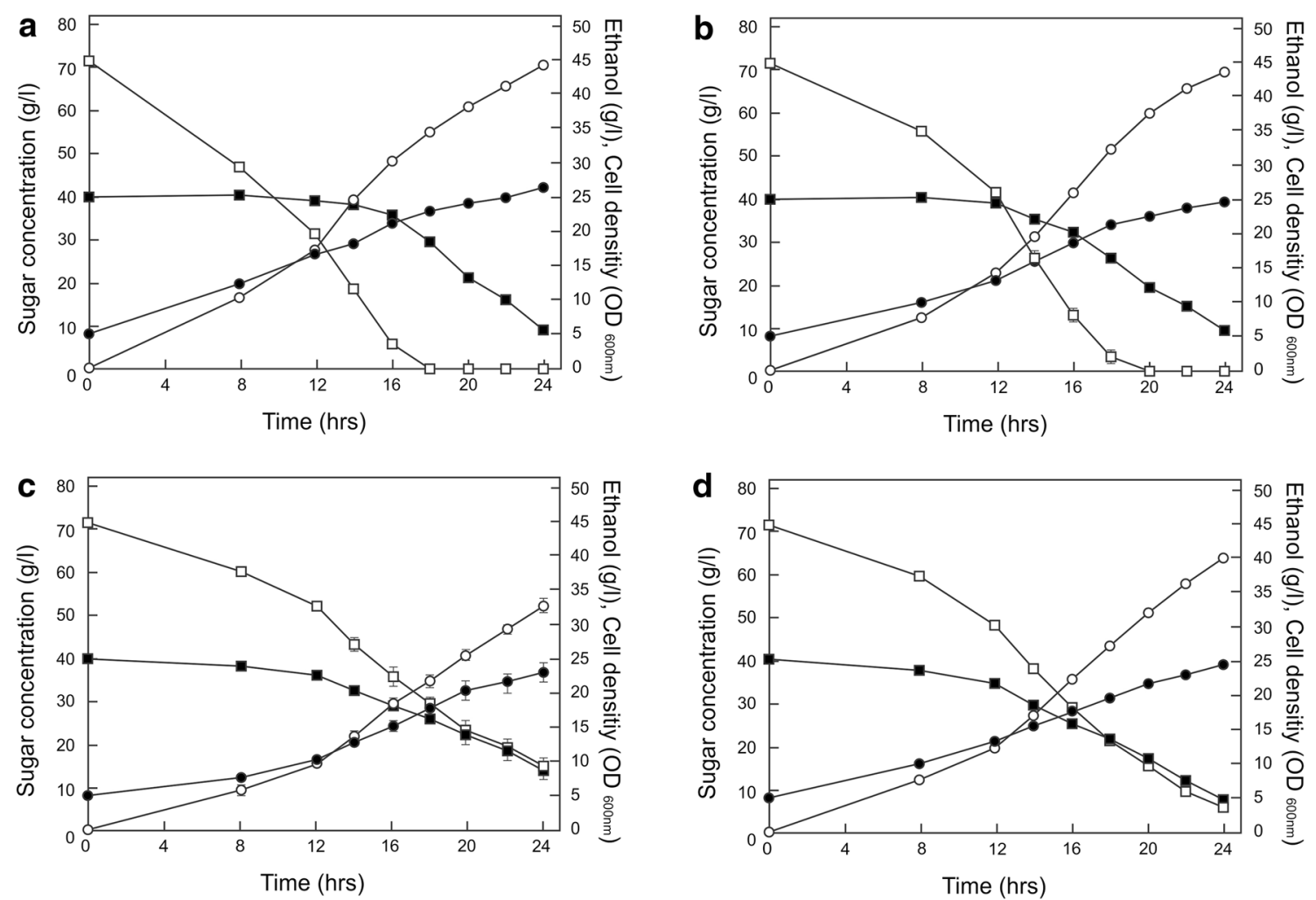

Fig. 3 Consumption of xylose and glucose by the control strain DS68616 containing the empty vector expressing all endogenous Hxt transporters a, and the transporter-deficient strain DS68625 expressing Hxt1 1 b, Hxt11-N366M c or Hxt1 1-N366T d, respectively. Symbols: glucose (open square), xylose (filled square), ethanol (open circle), and cell density (filled circle). The xylose and glucose concentration was 4 and $7 \%$. The error bars are the standard error of the mean from three independent experiments with triple samples

line with the rates observed in a strain using the full complement of native transporters.

Although the previously described Gal2-N376V and Hxt7-N370S mutants that localize with an improved affinity for D-xylose [28] localize in the same helical region, i.e., TMD 8, as with Hxt11 of this in this report, no co-consumption of glucose and xylose could be shown. This is likely because of the very low xylose transport rates by the mutant Hxt7 and Gal2 transporters compared to the Hxt11 transporter identified in this study. Also, the recently described Hxt7-N370S mutant still showed a higher affinity for glucose compared to xylose [28]. Recently, we also obtained mutants of Hxt36 with an improved D-xylose affinity [41]. In particular, the mutant Hxt36-N367A supported D-glucose and D-xylose co-consumption when both sugars are present in equimolar amounts. Also, the heterologous expression of xylose transporters Gxf1 from Candida intermedia, Sut1 from Pichia stipitis and At5g59250 from Arabidopsis thaliana was reported to improve xylose utilization by a recombinant $S$. cerevisiae $[12,16,38]$. For the Gxf1 transporter, the improvement of xylose utilization was restricted to substrate concentrations $>10 \mathrm{~g} / \mathrm{L}$ [12], whereas the Sut1 and At5g59250 transporters increased xylose uptake at substrate concentrations $>50 \mathrm{~g} / \mathrm{L}$ [16]. In addition, xylose-utilizing $S$. cerevisiae was not capable of producing ethanol at a low concentration of xylose $(<10 \mathrm{~g} / \mathrm{L})$, although the strain consumed xylose. During co-fermentation of xylose and glucose, the consumption rate of xylose is low as long as there is still glucose present in the growth medium [39]. Therefore, the present study demonstrates that Hxt11 is a more efficient transporter for ethanol production on sugar mixtures containing glucose and xylose as compared to the heterologously expressed specific xylose transporters mentioned above.

\section{Conclusions}

Here, we report for the first time that expression of the cryptic Hxt11 sugar transporter of S. cerevisiae stimulates xylose utilization both under aerobic and anaerobic conditions. Hxt11 has previously been overlooked as it is normally not expressed in S. cerevisiae. Xylose transport via Hxt11 is, however, less sensitive to glucose inhibition as compared to transport via the main 
hexose transporters in yeast such as Hxt2. The selectivity could further be enhanced by direct screening of Hxt11 mutants at position N366 for glucose-insensitive transport of xylose. When expressed in a sugar transporter-deficient strain of $S$. cerevisiae, selective mutants supported the co-consumption of xylose and glucose in mixed sugar fermentations using industrially relevant sugar concentrations. Because of the single transporter solution for co-consumption, future studies should be directed at increasing the rates of transport and metabolism to also reduce the duration of the fermentation. Our results demonstrate that endogenous transporter engineering is a powerful approach yielding a solution to a critical step that limits the use of yeast for the industrial fermentation of lignocellulosic biomass.

\section{Methods}

\section{Yeast strains and chemostat culture conditions}

S. cerevisiae strains used in this study (Table 1) were provided by DSM and may be made available for academic research under a strict Material Transfer Agreement with DSM (contact: paul.waal-de@dsm.com). The culture medium used was a defined mineral medium, with vitamins prepared as described by Verduyn et al. [40]. For storage of the strains, shake flask cultures were performed in the mineral medium supplemented with $2 \%$ maltose (Sigma-Aldrich) in case of the DS68616/ DS68625/DS68625-evo-derivatives, or $2 \%$ xylose (Roth) in case of DS71054-derivatives. With strains DS68625, DS68616, or DS71054, the Verduyn-urea was supplemented with $0.02 \%$ histidine (Sigma-Aldrich) to complement for the auxotrophic marker. Cultures were maintained at $30{ }^{\circ} \mathrm{C}$ in an orbital shaker until stationary growth phase was reached. After the addition of glycerol to $30 \%(\mathrm{v} / \mathrm{v})$, samples were stored in $2 \mathrm{ml}$ aliquots at $-80^{\circ} \mathrm{C}$.

Chemostat cultures were grown in a 3-L stirred tank bioreactor (Applikon, Schiedam, The Netherlands) filled with $500 \mathrm{ml}$ of the mineral medium at a temperature of $30{ }^{\circ} \mathrm{C}, \mathrm{pH} 4.5$ and at a dilution rate of $0.01-0.05 \mathrm{~h}^{-1}$. Stirring was performed at $400 \mathrm{rpm}$ and the starting $\mathrm{OD}_{600}$ was 1 . Some diffusion of oxygen was allowed via the inflowing medium and the venting vessel on the reactor.

\section{Hexose transporter deletion strain}

The eight major HXT (HXT1-7 and GAL2 [21]) genes were deleted to generate a xylose transport-negative model strain for screening. S. cerevisiae strain DS68616 equipped with DSM proprietary xylose fermentation technology based on Kuyper et al. [5, 7] was used for the following genetic modifications. Genes were deleted by standardly disrupting the target locus by integrating a floxed dominant resistance marker; markers were surrounded by loxP sites to allow for CRE-meditated marker removal and recycling [41]. Locus-specific flanks were 400-700 bp large and PCR-amplified using the primers listed in Additional file 2: Table S3. Up- and downstream flanks were fused to the floxed dominant resistance markers by standard cloning methods, and reamplified using the forward primer of the upstream flank (HXTxuf) and the reverse primer of the downstream flank (HXTxdr).

First, the HIS3 gene was deleted to serve as a marker for the introduction of putative enhanced xylose transporter(s) (libraries) as described below. The histidine auxotroph DS68616 was generated by the insertion of loxP-kanMX-loxP at the HIS3 locus. Subsequently, the marker was removed by CRE-mediated marker removal [41]. For the deletion of the eight main hexose transporter genes (HXT1-7 and GAL2) in S. cerevisiae, four deletion constructs were generated bearing either the kanMX, zeoMX, hphMX, nat MX [42, 43]. The hexose transporters were deleted in the following order: (1) HXT3-HXT6-HXT7 cluster, (2) HXT5-HXT1-HXT4 cluster, (3) GAL2, (4) HXT2. All transformations were plated on yeast extract $\left(10 \mathrm{~g} \mathrm{~L}^{-1}\right)$, peptone $\left(20 \mathrm{~g} \mathrm{~L}^{-1}\right)$ agar $(15 \mathrm{~g}$ $\mathrm{L}^{-1}$ ) medium supplemented with $20 \mathrm{~g} \mathrm{~L}^{-1}$ maltose. Maltose was added to the medium, because the uptake of this disaccharide occurs via an alternative transport system other than the Hxt glucose transporters [26]. With each deletion of a (cluster of) $H X T$ gene(s), an additional marker was inserted in the order: (1) hphMX, (2) nat MX, (3) zeo MX, (4) kanMX. With each inserted additional marker, the respective antibiotic was additionally supplemented to the medium in the following order: (1) HG, (2) HG and nour, (3) HG, nour and phleo, (4) HG, nour, phleo and G418. After integration of all four deletion constructs, a single colony was isolated under selection of all four antibiotics. The resulting strain, DS68625, did not grow on mineral medium (Verduyn-urea; based on Luttik et al. [44]) with $2 \%$ xylose, and showed a delayed growth pattern on Verduyn-urea with $2 \%$ glucose (data not shown). Correct integrations were verified by PCR analysis on genomic DNA isolates.

\section{Construction of hexokinase deletion strain}

A hexokinase deletion strain was constructed to allow screening for variants with improved ability to transport xylose in the competitive presence of glucose. Previously, a triple hexokinase mutant has been described to investigate the specificity for xylose transport $(\Delta h x k 1$, $\Delta h x k 2, \Delta g l k 1$; [45]). However, the additional deletion of galactokinase gene GAL1 is necessary since Gal1p can easily acquire SNPs enabling glucose conversion [35]. To prevent this, GAL1 was deleted. For deletion of hexokinase genes, oligonucleotides were designed (listed in 
Additional file 2: Table S4; numbers 834, 835, 838, 839, $846,847,848,849)$ comprised of 60 nucleotide flanking sequences homologous to the hexokinase gene locus, and of 20 nucleotides homologous to a floxed dominant resistance marker cassette (similar as described above except that kanMX was surrounded by lox66 and lox71). The oligonucleotides were used to amplify the deletion constructs. Subsequent PCR products were column filter-purified (GeneJet Kit, Thermo Fischer, Landsmeer, The Netherlands) and used for transformations experiments.

For the generation of a strain incapable of hexose metabolism but capable of hexose transport, four hexokinase gene deletions were made in the xylose-fermenting strain DS71055 (Table 1). Similar as in the DS68625 lineage, HIS3 was deleted to use as marker for the introduction of transporter constructs, similar as described for the other screening assay. The hexokinase and marker genes were deleted in the following order: (1) GLK1, (2) HXK1, (3) GAL1, (4) HXK2, (5) HIS3 integrating floxed kanMX, nat MX (followed by round of marker recycle), hphMX, kan MX, and nat MX (followed by round of marker recycle), respectively. After the deletion of GLK1 and $H X K 1$ both the integrated kanMX and nat MX marker were recycled by galactose-induced Cre-mediated recombination. After deletion of $H X K 2$, the intermediate strain was maintained on xylose-containing rich medium (YPX). After HIS3 deletion the integrated $h p h \mathrm{MX}$ and kanMX markers were removed by galactose-induced CRE recombination. To ensure growth of the strain, $2 \%$ xylose was added to YP $2 \%$ galactose + nourseothricin (YPGX). Selection on nourseothricin ensured maintenance of the natMX marker at the HIS3 locus leaving a selection trait to be used possibly later on. After single colony isolation, the strain was verified for its deletions and by colony PCR, and designated as DS71054. The final quadruple hexokinase mutant DS71054 was screened for growth on Verduyn-urea-his supplemented with $2 \%$ glucose or xylose. DS71054 did not grow on glucose, but did grow on xylose (Additional file 1: Figure S3). DS71054 was further maintained on YPX for storage and handling.

\section{Genomic DNA isolation, RNA extraction and cDNA synthesis}

Genomic DNA was isolated after $16 \mathrm{~h}$ of growth of the cells in the mineral medium containing $2 \%$ maltose using a modified yeast genomic DNA isolation protocol [46]. Yeast pellets from $2 \mathrm{ml}$ of exponential phase cell culture were mixed with $0.2 \mathrm{ml}$ of glass beads (diameter, $0.45 \mathrm{~mm}$ ) and disrupted in a Fastprep FP120 (Bio-101, Thermo Savant, California, USA) by a 45 -s burst at speed 6 .

Total RNA was isolated from the parental DS68625 and evolved DS68625-evo strains by a glass-bead disruption/
Trizol extraction procedure. Yeast pellets from $2 \mathrm{ml}$ of exponential phase cell culture were mixed with $0.2 \mathrm{ml}$ of glass beads (diameter, $0.45 \mathrm{~mm}$ ) and $0.9 \mathrm{ml}$ of Trizol with $125 \mu \mathrm{l}$ chloroform, and disrupted in a Fastprep FP120 (Bio-101, Thermo Savant, California, USA) by a 45-s burst at speed 6. Extracted total RNA (1 $\mu \mathrm{g})$ was converted into cDNA using the iScript Kit (Bio-Rad, US).

\section{Real-time PCR}

HXT8-HXT17 specific real-time PCR was performed using the SensiMix SYBR and Fluorescein kit (Quantace Ltd) and a MyiQ iCYCLER Real Time PCR instrument (Bio-Rad, Veenendaal, The Netherlands). Each 25- $\mu \mathrm{l}$ reaction contained $12.5 \mu$ l of SYBR green Master Mix, $4 \mu \mathrm{l}$ of extracted DNA, $0.5 \mu \mathrm{l}$ of each primer $(10 \mathrm{nM})$ and $7.5 \mu \mathrm{l}$ of sterile water. The PCR condition for HXT8-16 were $10 \mathrm{~min}$ at $95{ }^{\circ} \mathrm{C}$ followed by 39 cycles of amplification $\left(15 \mathrm{~s}\right.$ at $95^{\circ} \mathrm{C}, 30 \mathrm{~s}$ at $60^{\circ} \mathrm{C}, 30 \mathrm{~s}$ at $\left.72{ }^{\circ} \mathrm{C}\right)$. The primers shown in Additional file 2: Table S5 were utilized to amplify the mRNA fragments by PCR.

\section{Overexpression HXT11 and HXT12 in S. cerevisiae}

For PCR amplification, Phusion ${ }^{\circledR}$ High-Fidelity PCR Master Mix with HF buffer was used (Finnzymes; Fisher Scientific, Landsmeer, The Netherlands). Restriction enzymes and T4 DNA ligase were acquired from Fermentas (Fisher Scientific, Landsmeer, The Netherlands). The open-reading frames for HXT11 and HXT12 were PCRamplified from cDNA of the wild-type DS68625 using primers HXT11F (5'-GGCCTCTAGAATGTCAGGTGT TAATAATACATCCGC-3'), HXT12F (5'-GGCCTCTA GAATGGGTTTGATTGTCTCAATATTCAAC-3') and HXT11•12R (5'-CGATGGATCCTCAG CTGGAAAAGA ACCTCTTGTA AATTG-3'). The HXT11 and HXT12 PCR fragments were sequenced for validation, cut using

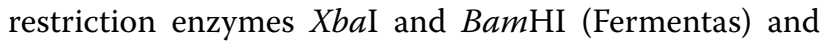
cloned in yeast expression vector pRS313-P7T7 behind the constitutive HXT7 (-391) promoter (P7) preceding the HXT7 terminator (T7). Plasmids were amplified and maintained in E. coli DH5 $\alpha$ cells. Plasmids were isolated from $E$. coli cultures using the GeneElute plasmid Miniprep kit (Sigma-Aldrich, The Netherlands). The HXT11 and HXT12 expression construct were transformed into S. cerevisiae DS68625 using standard yeast genetic techniques [47].

\section{Construction of HXT11-GFP expression plasmids}

The GFP gene in plasmid pDONR-eGFP-AT [48] was amplified by PCR with the primers GFP-F and GFP$\mathrm{R}$, and the product was cloned into the XmaI and ClaI restriction sites of pRS313-P7T7. The resulting plasmid and PCR products of the N366X mutants of HXT11 were digested with $X b a \mathrm{I}$ and $X m a I$, and ligated with the GFP 
cassette. HXT11-GFP chimeras were validated by DNA sequencing.

\section{Mutagenesis and screening for xylose transport mutants of Hxt11}

Error-prone PCR experiments were performed following the standard protocol [49] using DNA Taq polymerase (Thermo) with $10 \mathrm{ng}$ of template DNA in $100 \mu \mathrm{l}$ of PCR mix containing $5.5 \mathrm{mM} \mathrm{MgCl}_{2}$ and $0.15 \mathrm{mM} \mathrm{MnCl}_{2}$. The library of fragments was cloned into pRS313-P7T7 vector and transformed into the strain DS71054 that is unable to grow on glucose but that contains the xylose metabolic pathway. Three thousand variants were screened by growing the transformants on a 1:15 ratio of xylose $(1 \%)$ and glucose $(15 \%)$ with mineral medium using the 96-well plate format and Synergy MX (BioTek Instruments, Inc, USA) reader.

Saturated mutagenesis of position N366 in Hxt11 was done by PCR using Phusion ${ }^{\circledR}$ High-Fidelity PCR Master Mix with HF buffer using primer pairs F HXT11 XbaI/R HXT11 366NNN and F HXT11 366NNN/R HXT11 BamHI (Additional file 2: Table S6). The fragments of 1113 and 591 base pairs were subsequently used in an overlap PCR using the outside primers F HXT11 XbaI and R HXT11 BamHI and cloned into pRS313P7T7 using XbaI and BamHI. Sequencing of 48 E. coli clones yielded N367S (tct), N367P (cca), N367G (ggt), N367A (gcc), N367H (cac), N367R (cgc), N367L (ttg), N367C (tgt), N367T (acg), N367D (gat), N367Q (caa), and N367V (gtg). The remaining six amino acids at position 366 were amplified and cloned as mentioned above with overlap PCR using specific primers in which the NNN was replaced by ttt (F), gag (E), tgg (W), atg (M), aaa (K), and N367Y (tat).

\section{Deletion of the HXT11 gene and selection of knockout HXT11 strain}

For construction of the deletion plasmid pRS313-P7T7HIS (carrying loxP-his-loxP), the S. cerevisiae his gene including HXT11 promoter and terminator was amplified with oligonucleotides KOP11 (5'-AATAATCATTGCAC AATTTAGTTCTAAACGCTTTTGTTATTACTCAA TA T C C G T T T TA A G A G C T T G G T G A G C G C TAGGAGTC- $\left.3^{\prime}\right)$ and KOT11 (5'-TCGTCAATTTTT TTTTTTGCTTTTTTACCAATTTACCGAAAACTAG AAGAGAGTTCAAGAGAAAAAAAAAGAAAAA GCAAAAAGAAAAAAGGAAAGCGCGC- $3^{\prime}$ ) (Histidine gene underlined). The PCR fragment was transformed into the strain DS68625-evo and transformants were initially screen on $2 \%$ maltose plate for growth. Subsequently, to test growth differences of the HXT11 deletion in the DS68625-evo strain, cells were grown on $2 \%$ xylose for $72 \mathrm{~h}$ at $30{ }^{\circ} \mathrm{C}$, diluted to OD600 0.1 and grown to OD600 1.5-2 in 96-well plates. Genomic DNA was isolated from the yeast cells that were not able to grow on $2 \%$ xylose. To verify the deletion, the HXT11 gene was amplified with oligonucleotides delta-HXT11F (5'-ATGTCAGGTGTTAATAATACATCCGC-3') and delta-HXT11R (5'-TCAGCTGGAAAAGAACCTCTTG TAAATTG-3'), to yield a PCR fragment of $1704 \mathrm{bp}$. The PCR cycle was as follows: 35 cycles of denaturation at $98{ }^{\circ} \mathrm{C}$ for $10 \mathrm{~s}$, annealing at $65^{\circ} \mathrm{C}$ for $10 \mathrm{~s}$ and extension at $72{ }^{\circ} \mathrm{C}$ for $60 \mathrm{~s}$ followed by final extension for $10 \mathrm{~min}$ at $72{ }^{\circ} \mathrm{C}$.

\section{Uptake measurements}

The uptake of radiolabeled xylose or glucose was measured as follows: cells were collected by centrifugation (3000 rpm, $3 \mathrm{~min}, 20{ }^{\circ} \mathrm{C}$ ), washed and suspended into mineral medium. $\left[{ }^{14} \mathrm{C}\right]$ xylose or $\left[{ }^{14} \mathrm{C}\right]$ glucose (CAMPRO scientific $\mathrm{GmbH}$, Veenendaal, The Netherlands) stocks were added to the cells, uptake reactions were stopped at various time intervals by addition of $5 \mathrm{ml}$ of $0.1 \mathrm{M}$ lithium chloride, and the suspension was filtered $(0.45 \mu \mathrm{m} \mathrm{HV}$ membrane filter, Milipore, France). Filters were washed with another $5 \mathrm{ml}$ of lithium chloride and counted with the emulsifier scintillator plus (Perkin-Elmer, USA). Uptake experiments with strain DS68625 expressing Hxt11-variants were done with $0.5-500 \mathrm{mM}$ xylose or $0.1-500 \mathrm{mM}$ glucose. In general, the uptake of xylose was monitored after $1 \mathrm{~min}$ and for glucose after $15 \mathrm{~s}$ in quadruple. Glucose competition studies were performed with $50 \mathrm{mM}\left[{ }^{14} \mathrm{C}\right]$ xylose in the presence of $50-500 \mathrm{mM}$ unlabeled glucose.

\section{Molecular modeling}

The molecular modeling software package, including Prime 3.1 and Maestro 9.3, from Schrodinger LLC (NY, USA) was used to build a homology model for Hxt11. The crystal structure of XylE from E. coli with xylose bound (PDB ID: 4GBY) [37], which is $31.6 \%$ identical to Hxt11, was used as a template for the model building. Because of the lower identity, the sequence alignment between Hxt11 and XylE was manually corrected using information from family sequence alignments in the program Prime to accurately reflect the correct location of amino acid insertions and deletions.

\section{Fermentation}

Yeast cultures were grown in mineral medium containing $2 \%$ maltose for prepare inoculums for glucose and xylose fermentation experiments. Cells at mid-exponential phase were harvested and inoculated after washing twice by sterilized water. Fermentation experiments were performed using $100 \mathrm{ml}$ of mineral medium containing $7 \%$ glucose and $4 \%$ xylose in $120 \mathrm{ml}$ bottle at $30^{\circ} \mathrm{C}$ with initial $\mathrm{OD}_{600}$ of 5 under oxygen-limited conditions. All of the bottle fermentation experiments were repeated independently. 


\section{Fluorescence microscopy}

Plasmid pRS313-P7T7:GFP-HXT11 was transformed into $S$. cerevisiae strain DS68625. Individual colonies were used to inoculate minimal medium containing $2 \%$ maltose, and cells recovered from the exponential growth phase (at an optical density of 10 at $600 \mathrm{~nm}$ ) were subjected to fluorescence microscopy using a Nikon Eclipse-Ti microscope equipped with a $100 \times$ oil immersion objective, a filter set for GFP, and a Nikon DS-5Mc cooled camera (Nikon, Japan). Routinely, at least 100 cells per sample were examined, and each experiment was replicated at least three times.

\section{Miscellaneous analytical methods}

$S$. cerevisiae cells were grown in the mineral medium supplemented glucose and xylose with the initial $\mathrm{pH}$ adjusted to 4.5. Cell growth was monitored by optical density (OD) at $600 \mathrm{~nm}$ using UV-visible spectrophotometer. The concentration of glucose, xylose, and ethanol was measured by HPLC (Shimadzu, Kyoto, Japan) using an Aminex HPX-87H column (Bio-Rad, USA) and a refractive index detector (Shimadzu, Kyoto, Japan). The temperature of the column and detector was maintained at $65{ }^{\circ} \mathrm{C}$. The mobile phase was $0.005 \mathrm{~N} \mathrm{H}_{2} \mathrm{SO}_{4}$ at a flow rate of $0.55 \mathrm{ml} / \mathrm{min}$.

\section{Genomic analysis}

For genome sequencing (BaseClear Inc., Leiden, The Netherlands), genomic DNA of strain DS68625 and DS68625-evo was isolated to prepare for sequencing libraries using modified yeast genomic DNA isolation protocol [46]. The Illumina Miseq system was used with paired-end gDNA sequencing, and for analysis, the data were mapped to a reference genome (CEN.PK 113-7D) in Genebank. The results from the mapping were used to identify single-nucleotide polymorphism (SNPs) and insertions and deletions (INDELS) in the reads using the CLC Genomics Workbench (CLC bio).

\section{Additional files}

Additional file 1. Supplementary figures. Figure S1. Growth (O). glucose utilization $(\square)$ and ethanol production $(\mathbf{O})$ by the control strain DS68616 expressing all endogenous Hxt transporters and containing the empty vector (A), and the transporter-deficient strain DS68625 expressing Hxt11 (B). Figure S2. Xylose uptake by strain DS68625 expressing HXT2 (O) or HXT11 (O) in the presence of increasing glucose concentrations

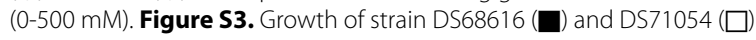
on $2 \%$ glucose (A) or $2 \%$ xylose (B). Figure S4. Maximum exponential growth rate of strain DS68625 expressing various Hxt11-N366X mutants on $2 \%$ glucose (A) or $2 \%$ xylose (B). The black bar indicated the wild-type position, and the gray bar indicates the N366D mutant obtained in the error-prone mutagenesis. The dashed line indicates the growth rate of strain DS68625 without any introduced transporter. The error bars are from two technical samples. Figure S5. Fluorescence images of strain DS68625 expressing GFP fusion proteins of Hxt11-N366X mutants grow on $2 \%$ maltose. Figure $\mathbf{S 6}$. Consumption of xylose and glucose at $1.0 \%$ each by the transporter-deficient strain DS68625 expressing Hxt11 (A) or Hxt1 1-N366T (B). Symbols: glucose ( $\square$ ), xylose $(\square)$, and ethanol (O). The error bars are from two technical samples. Figure S7. Ratio of the Xylose and glucose consumption rate in the presence of glucose. Strain DS68616 with the empty vector $(\mathbf{0})$, or strain DS68625 with a vector expressing Hxt1 1 (O), Hxt11 N366M ( $(\mathbf{\square})$ or Hxt1 1 N366T ( $\square$ ). Data calculated from the fermentation profiles presented in Fig. 3.

Additional file 2. Supplementary tables. Table S1. SNPs identified in the DS68625-evo strain by genome sequencing. Table S2. Fermentation profiles by the selected S. cerevisiae strain expressing Hxt11 and the Hxt11 N366M and N366T mutants during anaerobic batch cultivation. Table S3. Oligonucleotides used in hexose transporter strain construction. Table S4. Oligonucleotides used for construction hexokinase deletion strain. Table S5. Oligonucleotides used in qPCR. Table S6. Primers used for saturation mutagenesis of HXT11.

\section{Abbreviations}

GLK1: glucokinase; GAL1: galactokinase; HXK: hexokinase isozymes; HXT: hexose transporter; SNP: single-nucleotide polymorphism; SUMO: small ubiquitin-like modifier; TMD: transmembrane domain; PCR: polymerase chain reaction; XKS: xylulose kinase.

\section{Authors' contributions}

$H S, J N, P W$ and AD conceived and designed the research; HS performed the molecular biology and chemostat experiments and drafted the manuscript. $\mathrm{JN}$ participated in the design of the transporter mutant library and chemostat experiments. PW constructed the transporter and hexokinase deletion strains; RJ performed the molecular modeling experiments; PW, PK and AD supervised the project; the manuscript was written by contributions of all authors. All authors read and approved the final manuscript.

\section{Author details}

${ }^{1}$ Molecular Microbiology, Groningen Biomolecular Sciences and Biotechnology, University of Groningen, Zernike Institute for Advanced Materials and Kluyver Centre for Genomics of Industrial Fermentation, Nijenborgh 7, 9747 AG Groningen, The Netherlands. ${ }^{2}$ DSM Biotechnology Center, Alexander Fleminglaan 1,2613 AX Delft, The Netherlands.

\section{Acknowledgements}

The research has been financially supported by an EOS Long Term grant from the Dutch Ministry of Economical Affairs, Agriculture and Innovation, and by the research program of the biobased ecologically balanced sustainable industrial chemistry (BE-BASIC). We thank Viktor Boer (DSM) for critically reading the manuscript.

\section{Competing interests}

The authors declare they have no competing interests.

Received: 14 September 2015 Accepted: 22 October 2015

Published online: 02 November 2015

\section{References}

1. Ren C, Chen T, Zhang J, Liang L, Lin Z. An evolved xylose transporter from Zymomonas mobilis enhances sugar transport in Escherichia coli. Microb Cell Factories. 2009;8:66.

2. Zaldivar J, Nielsen J, Olsson L. Fuel ethanol production from lignocellulose: a challenge for metabolic engineering and process integration. Appl Microbiol Biotechnol. 2001;56:17-34.

3. Carroll A, Somerville C. Cellulosic biofuels. Annu Rev Plant Biol. 2009;60:165-82.

4. Bettiga M, Bengtsson O, Hahn-Hägerdal B, Gorwa-Grauslund MF. Arabinose and xylose fermentation by recombinant Saccharomyces cerevisiae expressing a fungal pentose utilization pathway. Microb Cell Factories. 2009;8:40. 
5. Kuyper M, Hartog MMP, Toirkens MJ, Almering MJH, Winkler AA, van Dijken JP, Pronk JT. Metabolic engineering of a xylose-isomerase-expressing Saccharomyces cerevisiae strain for rapid anaerobic xylose fermentation. FEMS Yeast Res. 2005;5:399-409.

6. Hamacher T, Becker J, Gárdonyi M, Hahn-Hägerdal B, Boles E. Characterization of the xylose-transporting properties of yeast hexose transporters and their influence on xylose utilization. Microbiol Read Engl. 2002;148(Pt 9):2783-8.

7. Kuyper M, Winkler AA, van Dijken JP, Pronk JT. Minimal metabolic engineering of Saccharomyces cerevisiae for efficient anaerobic xylose fermentation: a proof of principle. FEMS Yeast Res. 2004;4:655-64.

8. Demeke MM, Dietz H, Li Y, Foulquié-Moreno MR, Mutturi S, Deprez S, Den Abt T, Bonini BM, Liden G, Dumortier F, Verplaetse A, Boles $E$, Thevelein JM. Development of a D-xylose fermenting and inhibitor tolerant industrial Saccharomyces cerevisiae strain with high performance in lignocellulose hydrolysates using metabolic and evolutionary engineering. Biotechnol Biofuels. 2013;6:89.

9. Klinke HB, Thomsen AB, Ahring BK. Inhibition of ethanol-producing yeast and bacteria by degradation products produced during pre-treatment of biomass. Appl Microbiol Biotechnol. 2004;66:10-26.

10. Bellissimi E, van Dijken JP, Pronk JT, van Maris AJA. Effects of acetic acid on the kinetics of xylose fermentation by an engineered, xylose-isomerasebased Saccharomyces cerevisiae strain. FEMS Yeast Res. 2009;9:358-64.

11. Jeffries TW, Jin YS. Ethanol and thermotolerance in the bioconversion of xylose by yeasts. Adv Appl Microbiol. 2000;47:221-68.

12. Runquist D, Fonseca C, Rådström P, Spencer-Martins I, Hahn-Hägerdal B. Expression of the Gxf1 transporter from Candida intermedia improves fermentation performance in recombinant xylose-utilizing Saccharomyces cerevisiae. Appl Microbiol Biotechnol. 2009;82:123-30.

13. Young EM, Comer AD, Huang H, Alper HS. A molecular transporter engineering approach to improving xylose catabolism in Saccharomyces cerevisiae. Metab Eng. 2012;14:401-11.

14. Alper $\mathrm{H}$, Stephanopoulos $\mathrm{G}$. Engineering for biofuels: exploiting innate microbial capacity or importing biosynthetic potential? Nat Rev Microbiol. 2009;7:715-23.

15. Hahn-Hägerdal B, Galbe M, Gorwa-Grauslund MF, Lidén G, Zacchi G. Bio-ethanol-the fuel of tomorrow from the residues of today. Trends Biotechnol. 2006;24:549-56.

16. Hector RE, Qureshi N, Hughes SR, Cotta MA. Expression of a heterologous xylose transporter in a Saccharomyces cerevisiae strain engineered to utilize xylose improves aerobic xylose consumption. Appl Microbiol Biotechnol. 2008;80:675-84

17. Leandro MJ, Gonçalves P, Spencer-Martins I. Two glucose/xylose transporter genes from the yeast Candida intermedia: first molecular characterization of a yeast xylose-H+ symporter. Biochem J. 2006;395:543-9.

18. Bisson LF, Coons DM, Kruckeberg AL, Lewis DA. Yeast sugar transporters. Crit Rev Biochem Mol Biol. 1993;28:259-308.

19. Sedlak M, Ho NWY. Characterization of the effectiveness of hexose transporters for transporting xylose during glucose and xylose cofermentation by a recombinant Saccharomyces yeast. Yeast Chichester Engl. 2004;21:671-84.

20. Saloheimo A, Rauta J, Stasyk OV, Sibirny AA, Penttilä M, Ruohonen L. Xylose transport studies with xylose-utilizing Saccharomyces cerevisiae strains expressing heterologous and homologous permeases. Appl Microbiol Biotechnol. 2007;74:1041-52.

21. Reifenberger E, Freidel K, Ciriacy M. Identification of novel HXT genes in Saccharomyces cerevisiae reveals the impact of individual hexose transporters on glycolytic flux. Mol Microbiol. 1995;16:157-67.

22. van Maris AJA, Winkler AA, Kuyper M, de Laat WTAM, van Dijken JP, Pronk $J T$. Development of efficient xylose fermentation in Saccharomyces cerevisiae: xylose isomerase as a key component. Adv Biochem Eng Biotechnol. 2007;108:179-204.

23. Weierstall T, Hollenberg CP, Boles E. Cloning and characterization of three genes (SUT1-3) encoding glucose transporters of the yeast Pichia stipitis. Mol Microbiol. 1999;31:871-83.

24. Ozcan S, Johnston M. Function and regulation of yeast hexose transporters. Microbiol Mol Biol Rev MMBR. 1999;63:554-69.

25. Boles $E$, Hollenberg CP. The molecular genetics of hexose transport in yeasts. FEMS Microbiol Rev. 1997;21:85-111.
26. Wieczorke R, Krampe S, Weierstall T, Freidel K, Hollenberg CP, Boles E. Concurrent knock-out of at least 20 transporter genes is required to block uptake of hexoses in Saccharomyces cerevisiae. FEBS Lett. 1999;464:123-8.

27. Sikorski RS, Hieter P. A system of shuttle vectors and yeast host strains designed for efficient manipulation of DNA in Saccharomyces cerevisiae. Genetics. 1989;122:19-27.

28. Simpson-Lavy KJ, Johnston M. SUMOylation regulates the SNF1 protein kinase. Proc Natl Acad Sci USA. 2013;110:17432-7.

29. Forsburg SL, Guarente L. Mutational analysis of upstream activation sequence 2 of the CYC1 gene of Saccharomyces cerevisiae: a HAP2-HAP3responsive site. Mol Cell Biol. 1988;8:647-54.

30. Schüller H-J. Transcriptional control of nonfermentative metabolism in the yeast Saccharomyces cerevisiae. Curr Genet. 2003;43:139-60.

31. Nourani A, Wesolowski-Louvel M, Delaveau T, Jacq C, Delahodde A. Multiple-drug-resistance phenomenon in the yeast Saccharomyces cerevisiae: involvement of two hexose transporters. Mol Cell Biol. 1997;17:5453-60.

32. Salusjärvi L, Kankainen M, Soliymani R, Pitkänen J-P, Penttilä M, Ruohonen L. Regulation of xylose metabolism in recombinant Saccharomyces cerevisiae. Microb Cell Factories. 2008;7:18.

33. Johnston M. Feasting, fasting and fermenting. Glucose sensing in yeast and other cells. Trends Genet TIG. 1999;15:29-33.

34. Gancedo JM, Clifton D, Fraenkel DG. Yeast hexokinase mutants. J Biol Chem. 1977;252:4443-4.

35. Wisselink $\mathrm{H}$, Van Maris A, Pronk J. Pentose and glucose fermenting yeast cell. PCT Patent WO/2012/049170. April 19, 2012. http://www.directorypatent.com/WO/woZZSLASHZZ2012ZZSLASHZZ049170.html.

36. Farwick A, Bruder S, Schadeweg V, Oreb M, Boles E. Engineering of yeast hexose transporters to transport D-xylose without inhibition by D-glucose. Proc Natl Acad Sci USA. 2014;111:5159-64.

37. Sun L, Zeng X, Yan C, Sun X, Gong X, Rao Y, Yan N. Crystal structure of a bacterial homologue of glucose transporters GLUT1-4. Nature. 2012;490:361-6.

38. Runquist D, Hahn-Hägerdal B, Rådström P. Comparison of heterologous xylose transporters in recombinant Saccharomyces cerevisiae. Biotechnol Biofuels. 2010;3:5.

39. Lau MW, Dale BE. Cellulosic ethanol production from AFEX-treated corn stover using Saccharomyces cerevisiae 424A(LNH-ST). Proc Natl Acad Sci USA. 2009;106:1368-73.

40. Verduyn C, Postma E, Scheffers WA, Van Dijken JP. Effect of benzoic acid on metabolic fluxes in yeasts: a continuous-culture study on the regulation of respiration and alcoholic fermentation. Yeast Chichester Engl. 1992:8:501-17.

41. Güldener U, Heck S, Fielder T, Beinhauer J, Hegemann JH. A new efficient gene disruption cassette for repeated use in budding yeast. Nucleic Acids Res. 1996;24:2519-24.

42. Hansen J, Felding T, Johannesen PF, Piskur J, Christensen CL, Olesen K. Further development of the cassette-based pYC plasmid system by incorporation of the dominant hph, nat and AUR1-C gene markers and the lacZ reporter system. FEMS Yeast Res. 2003;4:323-7.

43. Steensma HY, Ter Linde JJ. Plasmids with the Cre-recombinase and the dominant nat marker, suitable for use in prototrophic strains of Saccharomyces cerevisiae and Kluyveromyces lactis. Yeast Chichester Engl. 2001;18:469-72.

44. Luttik MA, Kötter P, Salomons FA, van der Klei IJ, Van Dijken JP, Pronk $J$ T. The Saccharomyces cerevisiae ICL2 gene encodes a mitochondrial 2-methylisocitrate lyase involved in propionyl-coenzyme A metabolism. J Bacteriol. 2000;182:7007-13.

45. Subtil T, Boles E. Competition between pentoses and glucose during uptake and catabolism in recombinant Saccharomyces cerevisiae. Biotechnol Biofuels. 2012;5:14.

46. Harju S, Fedosyuk H, Peterson KR. Rapid isolation of yeast genomic DNA: Bust n'Grab. BMC Biotechnol. 2004;4:8.

47. Gietz RD, Woods RA. Yeast transformation by the LiAc/SS Carrier DNA/PEG method. Methods Mol Biol Clifton NJ. 2006;313:107-20.

48. Kovalchuk A, Weber SS, Nijland JG, Bovenberg RAL, Driessen AJM. Fungal $A B C$ transporter deletion and localization analysis. Methods Mol Biol Clifton NJ. 2012;835:1-16.

49. Cirino PC, Mayer KM, Umeno D. Generating mutant libraries using errorprone PCR. Methods Mol Biol Clifton NJ. 2003;231:3-9. 